J. Lake Sci.(湖泊科学), 2017, 29(4): 848-858

DOI 10. 18307/2017. 0408

(c) 2017 by Journal of Lake Sciences

\title{
鄱阳湖流域南昌市城市湖泊水体抗生素污染特征及生态风险分析
}

\author{
丁惠君 ${ }^{1,2}$, 钟家有 ${ }^{1 * *}$, 吴亦㴋 ${ }^{2}$, 张维昊 ${ }^{2,3 * *}$, 邹斌春 ${ }^{1}$, 楼 倩 $^{1}$, 杨 平 $^{1}$, 方媛瑗 ${ }^{1}$ \\ ( 1 : 江西省水利科学研究院江西省鄱阳湖水资源与环境重点实验室, 南昌 330029) \\ (2: 武汉大学资源与环境科学学院, 武汉 430072) \\ (3:湖北省水资源安全协同创新中心, 武汉 430072)
}

\begin{abstract}
摘 要: 采用固相萃取-超高效液相色谱一质谱法对南昌市 5 个城市湖泊——艾溪湖、瑶湖、青山湖、象湖和东西湖水体的 抗生素进行监测,并分析其污染来源和生态风险. 结果表明, 南昌市 5 个城市湖泊水体中四环素类、磺胺类、喹诺酮类、林 可霰素及大环内酯类 5 类抗生素的检出浓度范围分别为 $\mathrm{ND} \sim 6.3 、 \mathrm{ND} \sim 32.0 、 \mathrm{ND} \sim 97.2 、 \mathrm{ND} \sim 54.7$ 和 $\mathrm{ND} \sim 98.4 \mathrm{ng} / \mathrm{L}$; 红霉 素和林可霉素是 5 个城市湖泊水体中的主要抗生素类型; 相比于其他湖泊, 南昌市城市湖泊水体中主要检出抗生素浓度 总体处于中等水平, 其中恩诺沙星的检出浓度高于其他对比湖泊. 南昌市城市湖泊中 6 种主要抗生素的风险商均小于 0.1 , 表明均为最低生态风险; 5 个城市湖泊的主要生态风险因子分别是艾溪湖为红䨠素, 瑶湖为磺胺二甲嘧啶, 青山湖为 罗红霉素, 象湖为红霉素, 东西湖为磺胺嘧啶. 本研究可为南昌市城市湖泊水环境管理, 特别是新型污染物的环境管理提 供基础依据.
\end{abstract}

关键词: 抗生素; 新型污染物; 城市湖泊;水体;污染; 生态风险; 南昌市; 鄱阳湖

\section{Characteristics and ecological risk assessment of antibiotics in five city lakes in Nanchang City, Lake Poyang Catchment}

DING Huijun ${ }^{1,2}$, ZHONG Jiayou ${ }^{1 * *}$, WU Yixiao ${ }^{2}$, ZHANG Weihao ${ }^{2,3 * *}$, ZOU Binchun ${ }^{1}$, LOU Qian ${ }^{1}$, YANG Ping $^{1} \&$ FANG Yuanyuan

(1: Jiangxi Provincial Key Laboratory of Water Resources and Environment of Poyang Lake, Jiangxi Institute of Water Sciences, Nanchang 330029, P.R.China)

(2: School of Resource and Environmental Science, Wuhan University, Wuhan 430079, P.R.China)

(3: Hubei Provincial Collaborative Innovation Center for Water Resources Security, Wuhan 430072, P.R.China)

\begin{abstract}
Antibiotic concentrations in the five urban lakes in Nanchang City were monitored with methods of solid phase extraction and high performance liquid chromatography tandem mass spectrometry. The sources of antibiotics were analyzed and ecological risk of typical antibiotics on fish, invertebrates and algae were assessed using Risk Quotients ( $R Q s)$. Results showed that, the detected concentration range of tetracyclines, sulfonamides, quinolones, lincomycin and macrolides were ND-6.3 ng/L, ND-32.0 ng/L, ND-97.2 ng/L, ND-54.7 ng/L and ND-98.4 ng/L, respectively. Erythromycin and lincomycin were the main antibiotic types in the lakes. The antibiotic concentration detected in the five lakes were in the medium level compared with other lakes. The detected concentration of enrofloxacin was relatively higher than that in other lakes. $R Q s$ of the six main detected antibiotics were all less than 0.1 , indicating the lowest ecological risk. The main ecological risk factors of the five lakes were erythromycin, sulfadimidine, roxithromycin, erythromycin and sulfadiazine respectively in Lake Aixi, Lake Yao, Lake Qingshan, Lake Xiang and Lake East-west. This study provides basis for water environment management, especially for controlling emerging pollutants in such urban lakes. Keywords: Antibiotics; emerging pollutants; city lakes; water; pollution; ecological risk; Nanchang City; Lake Poyang

* 江西省水利科技项目 (KT201607)、水利部鄱阳湖水资源水生态环境研究中心开放基金项目 (ZXKT201507) 和水利 部科技推广项目 (TG1520) 联合资助. 2016-06-17 收稿;2016-09-29 收修改稿. 丁惠君 (1983 ), 女, 高级工程 师; E-mail:dingdinghuijun@163.com.

** 通信作者; E-mail:jiayou@ jxsl.gov.cn; zhangwh@ whu.edu.cn.
\end{abstract}


近年来, 在水环境中检出抗生素的报道不断引起关注 ${ }^{[1]}$, 中国首份抗生素排放与污染地图的公布更是 受到社会各界的高度关注 ${ }^{[2]}$. 中国是抗生素的生产和使用大国 ${ }^{[3]}$, 人畜使用的抗生素有一半以上以母体形 式排放到环境中 ${ }^{[2]}$. 环境中抗生素残留带来的细菌抗性及水生态毒性等生态风险问题得到持续研究 ${ }^{[4-9]}$. 2014 年 4 月 30 日,世界卫生组织 (WHO) 发布《抗菌素耐药: 全球监测报告》称“后抗生素” 时代正在逼近. Nature 杂志随后发表文章表明,抗生素耐药性问题正在发展中国家迅速曼延,抗生素耐药性问题的蔓延程度 远远超出我们的想象 ${ }^{[10]}$. 不仅如此, 已有的研究表明, 环境中抗生素残留对非目标生物也具有一定的毒性 作用: 如环丙沙星和磺胺甲恶唑会抑制海洋固着藻类和细菌的碳源利用 ${ }^{[11]}$ 、甲氧苄氨嘧啶降低了水虫肠道

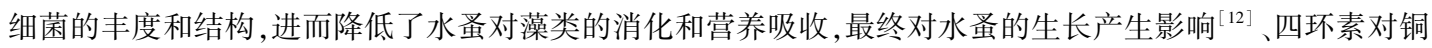
绿微囊藻和羊角月牙藻具有抑制光合作用系统和抗氧化系统的作用 ${ }^{[13]}$; 低质量浓度的磺胺嘧啶暴露, 可显 著促进斑马鱼的自主运动, 增大斑马鱼的心率, 斑马鱼胚胎在药物暴露处理过程中, 均产生畸形效应 ${ }^{\left[{ }^{[4]}\right.}$. 目 前, 国内外对环境抗生素残留的研究主要集中在河流 ${ }^{[15]}$ 、城市污水处理厂 ${ }^{[16]}$ 、水产养殖水体 ${ }^{[17]}$ 、医疗废 水 ${ }^{[18]}$ 以及沿海水体 ${ }^{[19]}$ 等. 我国对环境中抗生素残留的相关研究在 2005 年以后呈持续增加趋势, 然而对城 市湖泊抗生素残留的调查和风险评价等相关研究则相对较少. 城市湖泊具有防汛排涝、休闲娱乐、调节气候 以及改善城市生态环境等多种功能. 调查表明, 全球范围内 30\% 40\%的湖泊和水库已不同程度地受到富营 养化的影响 ${ }^{[20]}$. 城市湖泊接纳外源性营养盐的不断输人引发湖泊水体的富营养化, 而外源污染的输人也必 然伴随着与人畜排泄密切相关的抗生素污染的输人. 因此,城市湖泊水体抗生素污染问题已经成为当前环 境抗生素污染研究的重要内容, 然而相关研究报道却非常匮乏. 唐俊等 ${ }^{[21]}$ 的研究表明巢湖中 5 种磺胺类抗 生素的平均检出浓度为 $2.1 \sim 19.3 \mathrm{ng} / \mathrm{L}$, 最大检出浓度为 $137.9 \mathrm{ng} / \mathrm{L}$. 雷晓宁等 ${ }^{[22]}$ 的研究表明, 博斯腾湖环 丙沙星的平均浓度为 $39.22 \mathrm{ng} / \mathrm{L}$, 是该湖的主要抗生素.

南昌市是江西省的省会城市,地处江西省中部偏北, 赣江、抚河下游, 濒临中国第一大淡水湖——鄱阳 湖的西南岸. 2015 年, 南昌市的户籍人口达到 530 万, 人口密度达到 716 人 $/ \mathrm{km}^{2}$, 远高于全国平均人口密度 143 人 $\mathrm{km}^{2}$. 南昌市水网密布, 市区湖泊主要有青山湖、艾溪湖、象湖、东湖、西湖、南湖、北湖, 黄家湖、瑶湖 等, 城在湖中, 湖在城中. 随着城市化进程的不断发展, 南昌市城市湖泊水环境面临较大挑战, 如青山湖于 2008 年首次出现蓝藻, 2014 年 8 月青山湖暴发了历来最严重的蓝藻灾害 ${ }^{[23]}$. 本研究选择南昌市最主要的 5 个城市湖泊, 艾溪湖、瑶湖、青山湖、象湖和东南西北湖 (简称东西湖) 进行 5 类 18 种抗生素残留的监测研 究, 对 5 个湖泊检出的典型抗生素进行来源分析, 并运用风险商值法进行典型检出抗生素的生态风险评价, 以期为南昌市城市湖泊水环境管理提供基础依据.

\section{1 材料与方法}

\section{1 仪器与试剂}

抗生素标准品包括四环素类抗生素 $(\mathrm{TCs}) 、$ 、磺胺类抗生素 $(\mathrm{SAs})$ 、喹诺酮类抗生素 $(\mathrm{QUs})$ 、大环内酯类抗 生素 (MLs) 和林可霉素, 其种类及性质见表 1. 所有标准品均购自德国 Dr. Ehrenstorfer 公司. 甲醇和乙腈为 色谱纯, 购自美国 Tedia 公司;乙酸铵、甲酸和乙二胺四乙酸二钠 $\left(\mathrm{Na}_{2} \mathrm{EDTA}\right)$ 为分析纯, 购自上海国药集团化 学试剂有限公司.

UPLC, 1290, 美国 Agilent; 三重四极杆质谱,6460, 美国 Agilent; 固相萃取装置, 24 孔, 美国 SUPELCO; 氮 吹仪, DC-12,上海安谱; OasisHLB 小柱,6cc $500 \mathrm{mg}$,美国 Waters.

\section{2 样品采集及预处理}

2016 年 1 月, 对南昌市 $\left(28^{\circ} 09^{\prime} \sim 29^{\circ} 11^{\prime} \mathrm{N}, 115^{\circ} 27^{\prime} \sim 116^{\circ} 35^{\prime} \mathrm{E}\right)$ 的艾溪湖、瑶湖、青山湖、象湖和东西湖共 36 个点的表层水体 (水面下 $0.5 \mathrm{~m}$ ) 进行采集. 其中, 艾溪湖 8 个点 ( A1 A8)、瑶湖 9 个点 ( YH1 YH9)、青山 湖 9 个点 $(\mathrm{Q} 1 \sim \mathrm{Q} 9)$ 、象湖 4 个点 $(\mathrm{XH1} \sim \mathrm{XH} 4)$ 、东西湖 6 个点 (DH1、DH2、DH3、BH1、BH2、XH) (图 1). 水样 均用 $1 \mathrm{~L}$ 棕色玻璃瓶冷藏保存,并在 $48 \mathrm{~h}$ 内进行处理; 另取 $1 \mathrm{~L}$ 水样进行水体理化指标分析.

抗生素水样的固相萃取前处理参照文献 ${ }^{[25]}$. 固相萃取前, 准确量取 $1 \mathrm{~L}$ 水样, 用 $5 \mathrm{~mol} / \mathrm{L} \mathrm{H}_{2} \mathrm{SO}_{4}$ 调节 $\mathrm{pH}=3$, 并添加 $0.2 \mathrm{~g} \mathrm{Na} \mathrm{E}_{2} \mathrm{EDTA}$ 摇匀溶解, 再加人 $20 \mu \mathrm{l} 1 \mathrm{mg} / \mathrm{L}$ 的混合内标物以备内标法定量. 固相萃取 HLB 小柱依次经过 $10 \mathrm{ml}$ 甲醇和 $10 \mathrm{ml}$ 纯水活化处理, 水样以 $5 \sim 10 \mathrm{ml} / \mathrm{min}$ 的流速进过 HLB 小柱萃取, 再用 10 
$\mathrm{ml} \mathrm{5 \%}$ 甲醇淋洗, 用真空泵抽干 $2 \mathrm{~h}$, 最后用 $12 \mathrm{ml}$ 甲醇洗脱, 用氮吹仪吹干至 $0.5 \mathrm{ml}$, 再用超纯水定容至 1 $\mathrm{ml}$, 混匀待测.

表 1 抗生素标准品种类及性质

Tab.1 Standard antibiotics and their properties

\begin{tabular}{|c|c|c|c|c|c|c|}
\hline 抗生素类型 & 抗生素 & 缩写 & $\mathrm{CAS}$ 号 & 分子量 & $\operatorname{lgKow}^{\mathrm{a}}$ & $\begin{array}{l}\text { 溶解度/ } \\
(\mathrm{mg} / \mathrm{L})^{\mathrm{a}}\end{array}$ \\
\hline \multirow[t]{5}{*}{ 四环素类 } & 四环素 & $\mathrm{TC}$ & $60-54-8$ & 444.44 & -1.329 & 231 \\
\hline & 金霉素 & CTC & $57-62-5$ & 478.89 & -0.684 & 630 \\
\hline & 土霉素 & OTC & $79-57-2$ & 460.44 & -2.867 & 313 \\
\hline & 强力霉素 & DC & $564-25-0$ & 444.44 & -1.365 & 630 \\
\hline & 去甲金霉素 * & DTC & $127-33-3$ & 464.86 & -1.138 & - \\
\hline \multirow[t]{6}{*}{ 磺胺类 } & 磺胺嘧啶 & SD & $68-35-9$ & 250.28 & -0.338 & 77 \\
\hline & 磺胺吡啶 & SPD & $144-83-2$ & 249.29 & 0.527 & 268 \\
\hline & 磺胺甲恶唑 & SMX & $723-46-6$ & 253.28 & 0.484 & 610 \\
\hline & 磺胺噻唑 & $\mathrm{STZ}$ & $72-14-0$ & 255.32 & 0.715 & 373 \\
\hline & 磺胺二甲嘧啶 & SMZ & $57-68-1$ & 278.33 & 0.757 & 1500 \\
\hline & 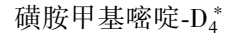 & SMR-D $_{4}$ & $127-79-7$ & 268.33 & 0.210 & 202 \\
\hline \multirow[t]{7}{*}{ 喹诺酮类 } & 双氟沙星 & DIF & $98106-17-3$ & 399.39 & 1.283 & 1333 \\
\hline & 诺氟沙星 & NFX & $70458-96-7$ & 319.33 & -0.306 & $9.439 \times 10^{4}$ \\
\hline & 氧氟沙星 & OFX & $82419-36-1$ & 361.37 & -0.200 & $1.081 \times 10^{4}$ \\
\hline & 环丙沙星 & CFX & $85721-33-1$ & 331.35 & -0.001 & $3 \times 10^{4}$ \\
\hline & 恩诺沙星 & EFX & $93106-60-6$ & 359.40 & 0.701 & 2007 \\
\hline & 依诺沙星 & ENX & $74011-58-8$ & 320.32 & -0.210 & $1.741 \times 10^{4}$ \\
\hline & 环丙沙星- $\mathrm{D}_{8}^{*}$ & CFX-D 8 & $1216659-54-9$ & 375.85 & - & - \\
\hline 林可霉素类 & 林可霉素 & LCM & $154-21-2$ & 406.54 & 0.288 & 92.19 \\
\hline \multirow[t]{3}{*}{ 大环内酯类 } & 罗红霉素 & RTM & $80214-83-1$ & 837.05 & 2.751 & 0.01887 \\
\hline & 红霉素 & ETM & $114-07-8$ & 733.93 & 2.478 & 4.239 \\
\hline & 红霉素- ${ }^{13} \mathrm{C}-\mathrm{D}_{3}{ }^{*}$ & ETM- ${ }^{13} \mathrm{C}-\mathrm{D}_{3}$ & $959119-26-7$ & 737.94 & - & - \\
\hline
\end{tabular}

* 表示内标物 $; \mathrm{a}$ 表示数据来源于美国环境保护局 $\mathrm{ECOSAR}$ 模型 ${ }^{[24]}$; 一表示数据缺失.

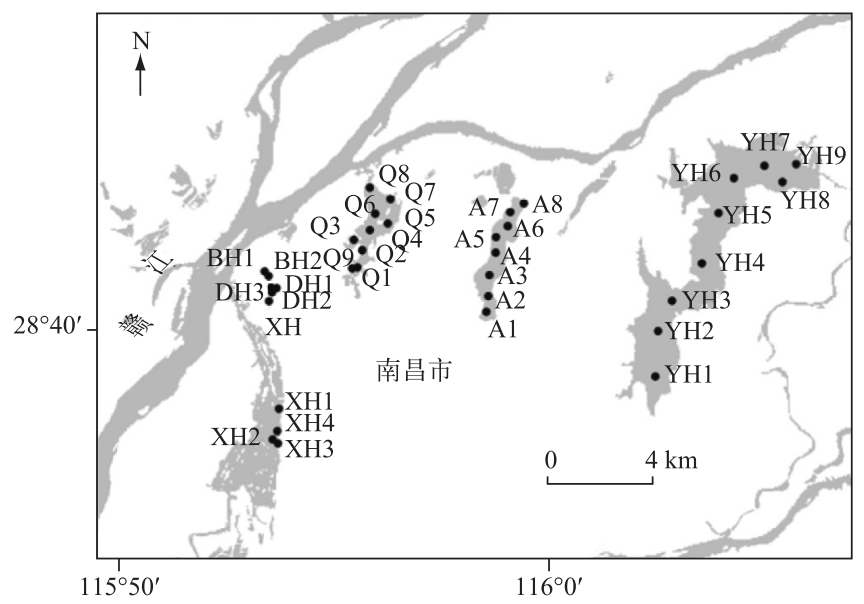

图 1 南昌市城市湖泊抗生素采样监测点位

Fig. 1 Sampling sites of antibiotics in five city lakes in Nanchang City 


\section{3 样品分析}

抗生素样品分析采用超高效液相色谱串联三重四级杆质谱 (UPLC-MS/MS) 进行测定, 测定方法参照前 期研究 ${ }^{[26-27]}$. 具体为,色谱柱采用 Eclipse Plus $\mathrm{C}_{18}$ 柱 $(100 \mathrm{~mm} \times 2.1 \mathrm{~mm}, 1.8 \mu \mathrm{m}$, Agilent $)$; 流速为 $0.3 \mathrm{ml} / \mathrm{min}$, 柱温为 $40^{\circ} \mathrm{C}$, 进样体积为 $10 \mu \mathrm{l}$; 流动相为 $0.2 \%$ 甲酸的 $2 \mathrm{mmol} / \mathrm{L}$ 乙酸铵溶液 $(\mathrm{A})$-乙腈 $(\mathrm{B})$. 采用梯度洗 脱程序为: 磺胺类: $0 \sim 0.1 \mathrm{~min}, 95 \% \sim 90 \% \mathrm{~A} ; 0.1 \sim 1.8 \mathrm{~min}, 90 \% \sim 90 \% \mathrm{~A} ; 1.8 \sim 2.8 \min , 90 \% \sim 87 \% \mathrm{~A} ; 2.8 \sim 6.9$ $\min , 87 \% \sim 51 \%$ A; $6.9 \sim 7.5 \min , 51 \% \sim 95 \%$ A. 四环素类: $0 \sim 0.1 \min , 95 \% \sim 92 \%$ A; $0.1 \sim 2.5 \min , 92 \% \sim$ $80 \%$ A $; 2.5 \sim 2.6 \min , 80 \% \sim 75 \%$ A $; 2.6 \sim 6.0 \min , 75 \% \sim 65 \%$ A $; 6.0 \sim 7.5 \min , 65 \% \sim 95 \%$ A. 喹诺酮类: $0 \sim 0.1$ $\min , 90 \% \sim 87 \% \mathrm{~A} ; 0.1 \sim 1.5 \mathrm{~min}, 87 \% \sim 87 \% \mathrm{~A} ; 1.5 \sim 8.0 \mathrm{~min}, 87 \% \sim 60 \% \mathrm{~A} ; 8.0 \sim 8.5 \mathrm{~min}, 60 \% \sim 90 \% \mathrm{~A}$. 大环内 酯类和林可霉素类:0 2 min, $90 \% \sim 65 \%$ A; $2.0 \sim 3.0 \mathrm{~min}, 65 \% \sim 40 \% \mathrm{~A} ; 3.0 \sim 5.0 \mathrm{~min}, 40 \% \sim 90 \% \mathrm{~A}$. 离子化模 式为 $\mathrm{ESI}^{+}$, 扫描模式为多重反应监测模式 (MRM), 干燥气温度 $325^{\circ} \mathrm{C}$, 干燥气流量 $6 \mathrm{~L} / \mathrm{min}$, 鞘气温度 $350^{\circ} \mathrm{C}$, 鞘气流量 $11 \mathrm{~L} / \mathrm{min}$, 雾化器压力 $45 \mathrm{psi}$. 各目标抗生素的母离子、子离子、碎裂电压、碰撞能和保留时间等参 数见表 2 .

表 218 种抗生素的色谱保留时间和质谱参数

Tab.2 Determination parameters of 18 target antibiotics by UPLC-MS/MS

\begin{tabular}{|c|c|c|c|c|c|}
\hline 抗生素 & 保留时间/min & 母离子/ $(\mathrm{m} / \mathrm{z})$ & 子离子/(m/z) ${ }^{1)}$ & 碎裂电压/V & 碰撞能/ $\mathrm{eV}^{2)}$ \\
\hline 磺胺嘧啶 & 3.368 & 251.0 & $155.9 / 92.0$ & 75 & $9 / 25$ \\
\hline 磺胺噻唑 & 3.966 & 256.0 & $156.0 / 92.1$ & 101 & $9 / 24$ \\
\hline 磺胺吡啶 & 4.190 & 250.0 & $155.9 / 108.0$ & 86 & $11 / 21$ \\
\hline 磺胺二甲嘧啶 & 5.297 & 279.0 & $185.9 / 124.0$ & 99 & $12 / 20$ \\
\hline 磺胺甲恶唑 & 6.417 & 254.0 & $155.9 / 107.9$ & 87 & $9 / 21$ \\
\hline 四环素 & 4.137 & 445.1 & $410.0 / 153.9$ & 118 & $15 / 31$ \\
\hline 金霉素 & 4.972 & 478.9 & $443.9 / 462.0$ & 107 & $12 / 18$ \\
\hline 土霉素 & 3.849 & 461.0 & $426.1 / 443.0$ & 111 & $15 / 7$ \\
\hline 强力霉素 & 5.263 & 445.1 & $428.0 / 153.9$ & 114 & $14 / 31$ \\
\hline 双氟沙星 & 5.324 & 400.0 & $382.0 / 356.1$ & 107 & $17 / 15$ \\
\hline 诺氟沙星 & 3.891 & 320.0 & $302.0 / 231.0$ & 115 & $16 / 35$ \\
\hline 氧氟沙星 & 3.952 & 362.0 & $318.0 / 261.0$ & 108 & $16 / 26$ \\
\hline 环丙沙星 & 4.115 & 332.0 & $314.0 / 231.0$ & 118 & $17 / 39$ \\
\hline 恩诺沙星 & 4.672 & 360.0 & $342.0 / 316.0$ & 109 & $19 / 16$ \\
\hline 依诺沙星 & 3.646 & 321.0 & $303.0 / 232.0$ & 109 & $18 / 36$ \\
\hline 林可電素 & 2.166 & 470.1 & $126 / 70.1$ & 126 & $29 / 74$ \\
\hline 罗红霉素 & 4.020 & 837.4 & $158 / 57.6$ & 152 & $29 / 15$ \\
\hline 红霉素 & 3.680 & 734.3 & $158 / 679.2$ & 173 & $34 / 18$ \\
\hline
\end{tabular}

1) 为了定性的准确性, 每个物质的质谱条件都设置了一个母离子对应两个子离子, 分别用于物质的定量和定性分析, /左 边子离子用于定量, /右边子离子用于定性, 即只有同时产生这 2 种子离子才能被认定为该物质. 2) 两组碰撞能分别对应 了子离子列的两组子离子, 即/左边对应定量离子,/右边对应定性离子.

\section{4 质量控制}

本研究采用内标法定量,方法检出限计算参照文献 [ 28], 方法定量下限采用 4 倍检出限确定. 使用纯水 分别配制 10 和 $100 \mathrm{ng} / \mathrm{L} 18$ 种抗生素的混合溶液 $1 \mathrm{~L}$, 测定 18 种抗生素的浓度. $10 \mathrm{ng} / \mathrm{L}$ 溶液进行 7 次实验, 用 来计算方法检出限和定量下限. 加标回收率计算参照《环境监测分析方法标准制修订技术导则》( HJ $168-$ $2010)$ 的规定, $100 \mathrm{ng} / \mathrm{L}$ 溶液进行 3 次测定, 用样品 3 次测定的平均值除以加标量来计算加标回收率. 每种抗 生素的回收率都在 70\% 130\% 之间, 相对标准偏差在 $0.06 \% \sim 13 \%$ 之间, 方法检出限在 $0.18 \sim 2.80 \mathrm{ng} / \mathrm{L}$ 之间 (表 3), 能够满足水环境中抗生素测定的要求. 
表 318 种抗生素的加标回收率、方法检出限和定量下限

Tab.3 Recovery, method detection limit and method quantity lower limit of 18 target antibiotics

\begin{tabular}{|c|c|c|c|c|}
\hline \multirow{2}{*}{ 抗生素 } & \multicolumn{2}{|c|}{ 加标回收率 } & \multirow{2}{*}{$\begin{array}{c}\text { 方法检出限/ } \\
(\mathrm{ng} / \mathrm{L})\end{array}$} & \multirow{2}{*}{$\begin{array}{c}\text { 方法定量下限/ } \\
(\mathrm{ng} / \mathrm{L})\end{array}$} \\
\hline & $10 \mathrm{ng} / \mathrm{L}$ & $100 \mathrm{ng} / \mathrm{L}$ & & \\
\hline 四环素 & $119.39 \pm 12.75$ & $82.91 \pm 11.64$ & 1.43 & 5.70 \\
\hline 金霉素 & $92.92 \pm 4.64$ & $87.90 \pm 9.45$ & 1.50 & 6.00 \\
\hline 土霉素 & $129.18 \pm 5.38$ & $82.69 \pm 9.65$ & 1.83 & 7.32 \\
\hline 强力霉素 & $85.14 \pm 4.82$ & $97.33 \pm 6.02$ & 2.80 & 11.2 \\
\hline 磺胺嘧啶 & $87.12 \pm 2.11$ & $85.08 \pm 7.23$ & 1.57 & 6.28 \\
\hline 磺胺吡啶 & $123.24 \pm 5.21$ & $107.01 \pm 4.79$ & 0.97 & 3.88 \\
\hline 磺胺甲恶唑 & $121.75 \pm 8.47$ & $111.60 \pm 2.08$ & 1.02 & 4.08 \\
\hline 磺胺噻唑 & $105.11 \pm 7.89$ & $93.85 \pm 2.48$ & 1.19 & 4.76 \\
\hline 磺胺二甲嘧啶 & $107.53 \pm 3.11$ & $86.37 \pm 10.82$ & 0.18 & 0.72 \\
\hline 双氟沙星 & $78.30 \pm 9.24$ & $118.94 \pm 7.47$ & 0.39 & 1.56 \\
\hline 诺氟沙星 & $76.97 \pm 9.88$ & $96.37 \pm 4.34$ & 1.70 & 6.80 \\
\hline 氧氟沙星 & $74.16 \pm 8.95$ & $121.60 \pm 4.99$ & 0.85 & 3.4 \\
\hline 环丙沙星 & $116.29 \pm 10.05$ & $78.63 \pm 7.30$ & 0.78 & 3.12 \\
\hline 恩诺沙星 & $83.57 \pm 4.10$ & $110.92 \pm 9.41$ & 0.84 & 3.36 \\
\hline 依诺沙星 & $78.07 \pm 1.88$ & $88.21 \pm 11.44$ & 0.82 & 3.28 \\
\hline 林可霉素 & $91.12 \pm 0.63$ & $86.34 \pm 10.83$ & 0.21 & 0.84 \\
\hline 红霉素 & $83.28 \pm 0.06$ & $80.55 \pm 2.54$ & 0.81 & 3.24 \\
\hline 罗红霉素 & $113.43 \pm 5.61$ & $84.23 \pm 7.72$ & 0.54 & 2.16 \\
\hline
\end{tabular}

\section{2 结果与讨论}

\section{1 南昌市城市湖泊水体抗生素检出浓度及水平}

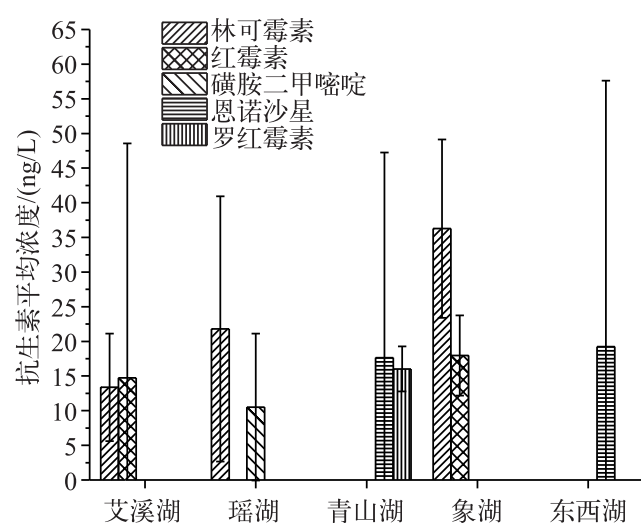

图 2 南昌市城市湖泊水体 主要检出抗生素浓度对比

Fig. 2 Concentration comparison of main detected antibiotics in water environment in five city lakes of Nanchang City
南昌市 5 个城市湖泊水体抗生素总体检出率及检 出浓度见表 4.5 个湖泊的 36 个采样点中, 检出率超过 $50 \%$ 的抗生素有红霉素 $61.11 \%$ 、磺胺嘧啶 $55.56 \%$ 、磺胺 二甲嘧啶 $52.78 \%$ 、罗红霉素 $52.78 \%$ 和林可霉素 $50.00 \%$. 最大检出浓度较高的抗生素有红需素 $98.40 \mathrm{ng} / \mathrm{L}$, 恩诺 沙星 $97.19 \mathrm{ng} / \mathrm{L}$, 林可震素 $54.71 \mathrm{ng} / \mathrm{L}$. 从检出率和检出 浓度来看, 红霉素和林可霉素是南昌城市湖泊中的主要 抗生素类型.

5 个城市湖泊中所含的主要抗生素类型依次是: 艾

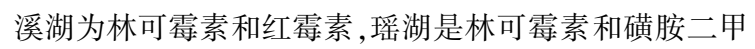
嘧啶, 青山湖是恩诺沙星和罗红霓素, 象湖是林可需素 和红霉素, 东西湖是恩诺沙星. 5 个湖泊水体 18 种目标 抗生素平均总浓度大小依次为象湖 $57.2 \mathrm{ng} / \mathrm{L}>$ 青山湖 $43.1 \mathrm{ng} / \mathrm{L}>$ 瑶湖 $38.5 \mathrm{ng} / \mathrm{L}>$ 艾溪湖 $32.3 \mathrm{ng} / \mathrm{L}>$ 东西湖 $25.6 \mathrm{ng} / \mathrm{L}$ (图 2). 5 个湖泊水体抗生素平均总浓度在同 一数量级, 按总浓度大小 5 个湖泊并没有呈现空间位置 分布上的规律性.

南昌市城市湖泊水体抗生素检出情况与其他湖泊的对比见表 5. 磺胺嘧啶在南昌市 5 个城市湖泊水体 中的检出浓度为 $\mathrm{ND} \sim 16.0 \mathrm{ng} / \mathrm{L}$, 与博斯腾湖 ${ }^{[22]}$ 和巢湖 ${ }^{[21]}$ 在一个数量级, 远小于白洋淀的最大检出浓度 505 $\mathrm{ng} / \mathrm{L}^{[37]}$. 磺胺二甲嘧啶在南昌城市湖泊水体中的检出浓度为 $\mathrm{ND} \sim 24.7 \mathrm{ng} / \mathrm{L}$, 与巢湖 ${ }^{[21]}$ 水平相当, 远小于太 
湖的最大检出浓度 $654.0 \mathrm{ng} / \mathrm{L}^{[36]}$. 恩诺沙星在南昌 5 个城市湖泊水体中的检出浓度为 $\mathrm{ND} \sim 97.2 \mathrm{ng} / \mathrm{L}$, 比太

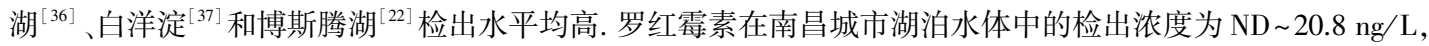
低于太湖 ${ }^{[36]}$ 和白洋淀 ${ }^{[37]}$ 最大检出浓度的 218.3 和 $155.0 \mathrm{ng} / \mathrm{L}$. 红霉素在南昌城市湖泊中的检出浓度为 $\mathrm{ND} \sim 98.4 \mathrm{ng} / \mathrm{L}$, 低于太湖 ${ }^{[36]}$ 和白洋淀 ${ }^{[37]}$ 的最大检出浓度 624.8 和 $121.0 \mathrm{ng} / \mathrm{L}$. 由此可见, 相比于其他相关湖 泊, 除恩诺沙星外, 南昌城市湖泊水体中几种主要检出抗生素的浓度总体处于中等水平, 恩诺沙星的检出浓 度则高于其他湖泊.

表 4 南昌市城市湖泊抗生素总体检出率及检出浓度 $(n=36)$

Tab.4 Overall detection frequencies and detection concentrations of 18 target antibiotics in city lakes of Nanchang City $(n=36)$

\begin{tabular}{|c|c|c|c|c|c|c|c|c|c|}
\hline & \multicolumn{4}{|c|}{ 四环素类 } & \multicolumn{5}{|c|}{ 磺胺类 } \\
\hline & 土霉素 & 四环素 & 金霉素 & 强力霉素 & 磺胺嘧啶 & 磺胺噻唑 & 磺胺吡啶 & $\begin{array}{l}\text { 磺胺二 } \\
\text { 甲嘧啶 }\end{array}$ & $\begin{array}{c}\text { 磺胺甲 } \\
\text { 恶唑 } \\
\end{array}$ \\
\hline 检出率 & $19.44 \%$ & $5.56 \%$ & $19.44 \%$ & $5.56 \%$ & $55.56 \%$ & $5.56 \%$ & $2.78 \%$ & $52.78 \%$ & $2.78 \%$ \\
\hline \multirow[t]{3}{*}{ 浓度范围/(ng/L) } & $\mathrm{ND}^{\mathrm{a}} \sim<M D L^{\mathrm{b}}$ & $\mathrm{ND} \sim<M D L$ & $\mathrm{ND} \sim 6.3$ & $\mathrm{ND} \sim<M D L$ & $\mathrm{ND} \sim 16.0$ & $\mathrm{ND} \sim 9.0$ & $\mathrm{ND} \sim 5.3$ & $\mathrm{ND} \sim 32.0$ & $\mathrm{ND} \sim<M D L$ \\
\hline & 林可霉素类 & \multicolumn{2}{|c|}{ 大环内酯类 } & \multicolumn{6}{|c|}{ 喹诺酮类 } \\
\hline & 林可霉素 & 罗红霉素 & 红霉素 & 依诺沙星 & 诺氟沙星 & 氧氟沙星 & 环丙沙星 & 恩诺沙星 & 双氟沙星 \\
\hline 检出率 & $50.00 \%$ & $52.78 \%$ & $61.11 \%$ & $38.89 \%$ & $16.67 \%$ & $2.78 \%$ & $13.89 \%$ & $27.78 \%$ & 0 \\
\hline 浓度范围/ (ng/L) & ND 54.7 & ND 20.8 & ND 98.4 & $\mathrm{ND} \sim 14.5$ & $\mathrm{ND} \sim 22.8$ & $\mathrm{ND} \sim<M D L$ & $\mathrm{ND} \sim 5.3$ & ND 97.2 & ND \\
\hline
\end{tabular}

a 表示无检出; b 表示方法定量限.

表 5 南昌市城市湖泊与其他湖泊抗生素检出水平对比 $(\mathrm{ng} / \mathrm{L})$

Tab.5 Concentration comparison of antibiotics in city lakes of Nanchang City and other lakes

\begin{tabular}{lccccccc}
\hline 湖泊 & 磺胺嘧啶 & 磺胺二甲嘧啶 & 恩诺沙星 & 林可霉素 & 罗红霉素 & 红需素 & 参考文献 \\
\hline 艾溪湖 & $\mathrm{ND} \sim 15.7$ & $<M D L \sim 1.0$ & $\mathrm{ND}$ & $\mathrm{ND} \sim 25.8$ & $\mathrm{ND} \sim 16.4$ & $<M D L \sim 98.4$ & 本研究 \\
瑶湖 & $<M D L \sim 16.0$ & $1.9 \sim 24.7$ & $\mathrm{ND}$ & $\mathrm{ND} \sim 54.7$ & - & $\mathrm{ND} \sim<M D L$ & 本研究 \\
青山湖 & $<M D L \sim 8.8$ & $\mathrm{ND} \sim<M D L$ & $\mathrm{ND} \sim 83.5$ & $\mathrm{ND}$ & $11.2 \sim 20.8$ & $\mathrm{ND} \sim<M D L$ & 本研究 \\
象湖 & $<M D L$ & $\mathrm{ND}$ & $\mathrm{ND} \sim 7.2$ & $19.2 \sim 49.7$ & $\mathrm{ND}$ & $11.3 \sim 24.5$ & 本研究 \\
东西湖 & $\mathrm{ND} \sim 10.3$ & $\mathrm{ND} \sim 2.4$ & $\mathrm{ND} \sim 97.2$ & $\mathrm{ND}$ & $\mathrm{ND}$ & $<M D L \sim 11.1$ & 本研究 \\
太湖 & - & $\mathrm{ND} \sim 654.0$ & $\mathrm{ND}$ & - & $\mathrm{ND} 218.3$ & $\mathrm{ND} \sim 624.8$ & {$[36]$} \\
白洋淀 & $0.86 \sim 505$ & - & $\mathrm{ND} \sim 4.42$ & - & $\mathrm{ND} \sim 155$ & $\mathrm{ND} \sim 121$ & {$[37]$} \\
博斯腾湖 & $2.88 \sim 37.27$ & - & $\mathrm{ND} \sim 15.22$ & - & - & - & {$[22]$} \\
巢湖 & $\mathrm{ND} \sim 45.6$ & $\mathrm{ND} \sim 4.7$ & - & - & - & - & {$[21]$} \\
\hline
\end{tabular}

一表示未检出.

\section{2 南昌市城市湖泊水体抗生素来源分析}

5 个城市湖泊中, 青山湖和东西湖均位于南昌市老城区中心区域, 艾溪湖和瑶湖位于南昌市东部城区, 象湖位于南昌市西南面. 本研究中, 青山湖 ( Q1 和 Q9) 的恩诺沙星和罗红霉素平均浓度相对较高, 东西湖 (DH1) 的恩诺沙星浓度相对较高. 恩诺沙星是我国畜牧业生产中常用的抗菌药 ${ }^{[29]}$, 青山湖西南部采样点 Q1 和 Q9 中, Q1 采样点为城市河玉带河的人口处, Q9 采样点为涨水期市政排水出口处, 这可能是由于 2 个采样 点含有养殖污染源以及市政污水的排人, 导致检出恩诺沙星和罗红霉素. 这与青山湖湖体水质北部最好、中 部次之、南部最差, 西部水质较差、东部水质较好的结论基本一致 ${ }^{[23]}$. 而东西湖的 DH1 采样点由于靠近养鱼 池导致恩诺沙星有检出.

艾溪湖位于南昌市城东、高新产业区内, 艾溪湖东岸为艾溪湖湿地公园. 2006 年, 艾溪湖经过综合整治 
后周边已没有排污口, 其唯一来水为南部的幸福渠 ( $\mathrm{A} 1$ 采样点附近). 研究表明 ${ }^{[30]}$, 每天经幸福渠进人艾溪 湖的水量大约为 $32 \times 10^{4} \mathrm{t}$, 主要包括城东地区 23 个村镇约 18 万人的生活污水, 昌东工业园区 200 多家企业 的生产废水, 以及当地畜禽养殖废水等. 而生活污水、养殖废水等是水环境中抗生素污染的主要来源 ${ }^{[1,31]}$. 由此可见, 由人、畜排放的废污水携带抗生素残留经幸福渠排人艾溪湖南岸, 进而扩散至整个湖区, 这是导

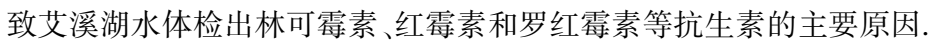

瑶湖位于南昌市东部城郊结合区, 该湖自南向北分为上瑶湖、中瑶湖和下瑶湖. 上瑶湖位于瑶湖最南 端, 周边有高校园区、氨厂、水产养殖基地和国际水上运动中心, 污染源较多; 中瑶湖周边以农田分布为主, 下瑶湖在瑶湖最北端, 污染源较少 ${ }^{[32]}$. 瑶湖水体中检出的主要抗生素为林可霉素和磺胺二甲嘧啶, 其中林 可霉素为人类常用抗生素, 磺胺二甲嘧啶是传统应用的抗菌药和抗球虫药, 在中国曾广泛用于畜禽的球虫 病. 在瑶湖所有的采样点中, 上瑶湖采样点 $(\mathrm{YH} 1 \sim \mathrm{YH} 3)$ 的抗生素检出浓度较大, 其次是中瑶湖和下瑶湖, 这 与其污染输人来源及其扩散方向较为一致.

象湖位于南昌市的西南角, 进入 21 世纪以来, 象湖周边的房地产业、工业、第三产业发展迅速, 对象湖 水质产生了不利影响 ${ }^{[33]}$; 另外, 象湖污水处理厂紧邻象湖北面, 污水处理厂出水排人象湖. 研究表明, 污水 处理厂的传统工艺对抗生素的降解去除能力有限, 大部分抗生素经污水处理厂处理后难以充分降解去除, 最终排人了受纳水体 ${ }^{[34]}$. 由此可见, 象湖周边外源性污染输人主要以周边居民生活污水为主. 本次研究象 湖采样点主要分布在象湖东面 (XH1 XH4), 检出的主要抗生素种类为林可霉素和红霉素, 而林可霉素和大 环内酯类抗生素主要用于人类治疗 ${ }^{[35]}$, 因此, 可以推断象湖采样点检出的主要抗生素种类与其周边生活污 水排放密切相关.

\section{3 南昌市城市湖泊抗生素生态风险评价}

环境中抗生素残留属于新型有机污染物, 其在环境中的浓度较常规污染物低一般为 $\mathrm{ng} / \mathrm{L}$ 级 $\sim \mu \mathrm{g} / \mathrm{L}$ 级. 目前,地表水环境中抗生素残留还没有相关的标准予以规范和约束. 国内外学者在评价环境药物残留的生 态风险时, 一般采用欧盟的技术指导文件 (TGD) 中关于环境风险评价的方法 ${ }^{[38]}$, 即采用风险商值 (risk quotients, $R Q s)$ 法, 计算公式为:

$$
R Q s=M E C / P N E C
$$

式中, $M E C$ 为污染物实际监测浓度 $(\mathrm{ng} / \mathrm{L}), P N E C$ 为预测无效应浓度 $(\mathrm{ng} / \mathrm{L}) . P N E C$ 一般通过有机物的无 观察效应浓度 $(N O E C)$ 除以评估因子获得. 由于目前缺乏大多数化合物的 $N O E C$, 生态风险评价中所用到的 $N O E C$ 需用从急性毒性数据或慢性毒性数据除以某个评估因子外推获得 $P N E C^{[39]}$.

本文采用 $\mathrm{ECOSAR}^{[40]}$ 模型提供的有机物对水生态系统中 3 类典型受试物种 (鱼、水蚤、藻) 的急性毒性 数据除以评估系数 1000 得到 $P N E C$, 即 $P N E C=E C_{50} / 1000^{[41]}$. 经计算, 得到磺胺嘧啶、磺胺二甲嘧啶、恩诺沙 星、林可霉素、罗红霉素及红䨚素 6 种抗生素对鱼、水蚤和藻类的 $P N E C$ 分别为 $1.516 、 0.002$ 和 $0.010 \mathrm{mg} / \mathrm{L}$; $0.291 、 0.002$ 和 $0.006 \mathrm{mg} / \mathrm{L} ; 4.923 、 0.505$ 和 $0.561 \mathrm{mg} / \mathrm{L} ; 1.040 、 0.102$ 和 $0.124 \mathrm{mg} / \mathrm{L} ; 0.052 、 0.007$ 和 $0.005 \mathrm{mg} / \mathrm{L}$; $0.068 、 0.009$ 和 $0.006 \mathrm{mg} / \mathrm{L}$.

按照 Hernando 等 ${ }^{[42]}$ 提出的 $R Q s$ 分类方法表征生态风险的不同程度: $R Q s<0.1$ 为最低风险, $0.1 \leqslant R Q_{s}<1$ 为中等风险, $R Q s \geqslant 1$ 为高风险. 由此, 本研究得到南昌市城市湖泊 6 种主要抗生素磺胺嘧啶、磺胺二甲嘧啶、 恩诺沙星、林可需素、罗红電素和红震素风险商如图 3 所示.

南昌市城市湖泊水体中 6 种主要抗生素的风险商均小于 0.1 , 其中除个别点位 (A1) 的红霉素对藻和水 蚤的 $R Q s>0.01$ 外, 其余均小于 0.01 , 表明均为最低生态风险 (图 3). 然而, 不同湖泊的各种抗生素风险水平 表现各异: 瑶湖中磺胺类抗生素 (磺胺嘧啶和磺胺二甲嘧啶) 的生态风险相对较高; 恩诺沙星在青山湖和东 西湖的生态风险相对较高; 林可霰素在艾溪湖、瑶湖和象湖的生态风险相对较高; 罗红霉素在艾溪湖和青山 湖的生态风险相对较高; 红霉素在艾溪湖和象湖的生态风险相对较高. 基于本研究确定的 5 类 18 种常见抗 生素, 取各湖泊水体中抗生素风险相对最高者为其主要抗生素生态风险因子, 得出 5 个湖泊的主要生态风 险因子分别是艾溪湖为红霉素, 瑶湖为磺胺二甲嘧啶, 青山湖为罗红霉素, 象湖为红電素, 东西湖为磺胺 嘧啶.

在 3 种模式水生生物中, 6 种抗生素对鱼类的生态风险水平相对最低, 而大环内酯类 (罗红霉素和红霉 

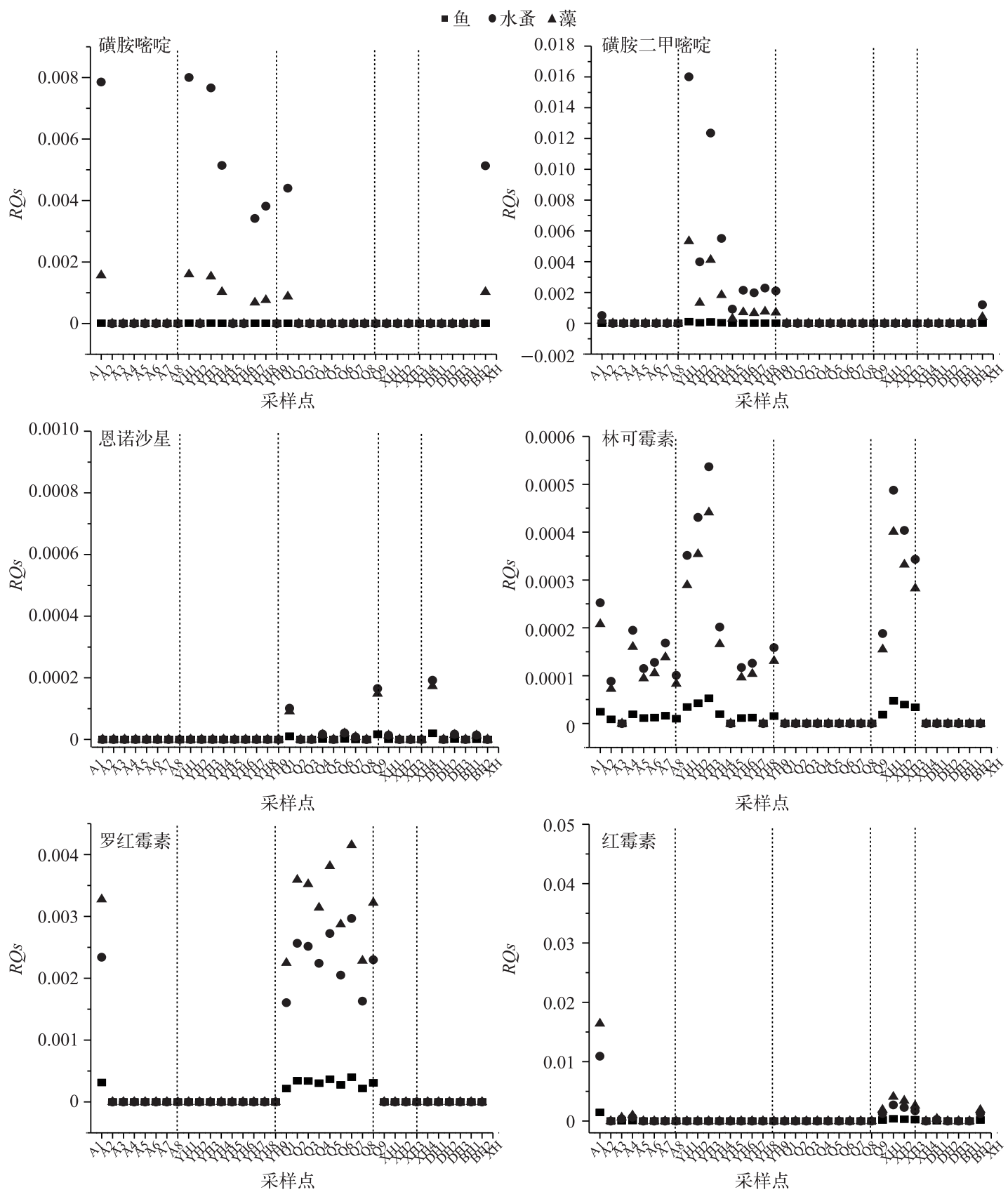

图 3 南昌市城市湖泊主要抗生素的风险商

Fig.3 RQs of main detected antibiotics in Nanchang City lakes

素) 对藻类的生态风险水平相对最高, 磺胺二甲嘧啶对水蚤的生态风险水平相对较高, 其余几种抗生素对水 蚤和藻类的生态风险水平相当,均高于对鱼类的生态风险水平. 这提示在由不同生态风险因子控制的湖泊 中, 面临风险的水生生物并不相同, 如艾溪湖、青山湖和象湖 3 个湖泊中藻类受到更大的生态风险, 瑶湖和 东西湖则是水蚤面临相对较大的生态风险. 尽管本文计算得到的抗生素生态风险水平并不高,但由于风险 商值法仅为针对单种污染物进行简单的毒性实验得到的评价结果, 而实际环境中存在着复合抗生素污染, 
以及抗生素与其他类型污染物的协同作用, 因此, 风险商值法可能会低估污染物的实际生态风险. 今后, 更 加全面的环境监测以及考虑复合污染的生态风险评价非常必要.

\section{3 结论}

1) 从检出率和检出浓度两方面看, 红霉素和林可霉素是南昌城市湖泊中的主要抗生素类型. 5 个城市 湖泊水体抗生素平均总浓度在同一数量级, 且并没有呈现空间位置分布上的规律性. 相比于其他相关湖泊, 南昌城市湖泊水体中几种主要检出抗生素的浓度总体处于中等水平, 恩诺沙星的检出浓度则高于其他湖泊.

2) 5 个城市湖泊水体抗生素检出结果与其污染输人来源基本一致.

3) 南昌市城市湖泊中 6 种主要抗生素的风险商均小于 0.1 , 表明均为最低生态风险; 5 个湖泊的主要生 态风险因子分别是艾溪湖为红震素, 瑶湖为磺胺二甲嘧啶, 青山湖为罗红需素, 象湖为红霉素, 东西湖为磺 胺嘧啶.

4) 在 3 种模式水生生物中, 6 种抗生素对鱼类的生态风险相对最低, 而大环内酯类 (罗红霉素和红雷 素) 对藻类的生态风险相对最高, 磺胺二甲嘧啶对水蚤的生态风险水平相对较高, 这提示在由不同生态风险 因子控制的湖泊中,面临风险的水生生物并不相同.

5) 风险商值法可能会低估污染物的实际生态风险, 更加全面的环境监测以及考虑复合污染的生态风险 评价非常必要.

\section{4 参考文献}

[ 1 ] Zhang Qiang, Xin Qi, Zhu Jingmin et al. The antibiotic contaminations in the main water bodies in China and the associated environmental and human health impacts. Environmental Chemistry, 2014, (7):1075-1083. [章强, 辛琦, 朱静敏等. 中国主要水域抗生素污染现状及其生态环境效应研究进展. 环境化学, 2014, (7) : 1075-1083.]

[ 2 ] Zhang QQ, Ying GG, Pan CG et al. Comprehensive evaluation of antibiotics emission and fate in the river basins of China: Source analysis, multimedia modeling, and linkage to bacterial resistance. Environmental Science \& Technology, 2015,49 (11) : 6772-6782.

[ 3 ] Richardson BJ, Larn PKS, Martin M. Emerging chemicals of concern: Pharmaceuticals and personal care products (PPCPs) in Asia, with particular reference to Southern China. Marine Pollution Bulletin, 2005, 50(9) : 913-920.

[ 4 ] Reardon S. Antibiotic resistance sweeping developing world. Nature, 2014, 509(7499) : 141-142.

[ 5 ] Johansson CH, Janmar L, Backhaus T. Toxicity of ciprofloxacin and sulfamethoxazole to marine periphytic algae and bacteria. Aquatic Toxicology, 2014, 156: 248-258.

[ 6 ] Gorokhova E, Rivetti C, Furuhagen S et al. Bacteria-mediated effects of antibiotics on daphnia nutrition. Environmental Science \& Technology, 2015, 49(9) : 5779-5787.

[ 7 ] Kong Weidong, Zhu Yongguan. A review on ecotoxicology of veterinary pharmaceuticals to plants and soil microbes. Asian Journal of Ecotoxicology, 2007, 2(1): 1-9. [孔维栋, 朱永官. 抗生素类兽药对植物和土壤微生物的生态毒理学效 应研究进展. 生态毒理学报, 2007, 2(1) : 1-9.]

[ 8 ] Zhou Qixing, Luo Yi, Wang Meie. Environmental residues and ecotoxicity of antibiotics and their resistance gene pollution: A review. Asian Journal of Ecotoxicology, 2007, 2(3): 243-251. [ 周启星, 罗义, 王美娥. 抗生素的环境残留、生 态毒性及抗性基因污染. 生态毒理学报, 2007, 2(3) : 243-251.]

[ 9 ] Gao Lihong, Shi Yali, Li Wenhui et al. Environmental behavior and impacts of antibiotics. Environmental Chemistry, 2013, 32(9) : 1619-1633. [高立红, 史亚利, 厉文辉等. 抗生素环境行为及其环境效应研究进展. 环境化学, 2013, 32(9) : 1619-1633.]

[10] Reardon S. Antibiotic resistance sweeping developing world. Nature, 2014, 509: 141-142.

[11] Johansson CH, Janmar L, Backhaus T. Toxicity of ciprofloxacin and sulfamethoxazole to marine periphytic algae and bacteria. Aquatic Toxicology, 2014, 156: 248-258.

[12] Gorokhova E, Rivetti C, Furuhagen S et al. Bacteria-mediated effects of antibiotics on daphnia nutrition. Environmental Science \& Technology, 2015, 49: 5779-5787.

[13] Yang W, Tang Z, Zhou F et al. Toxicity studies of tetracycline on Microcystis aeruginosa and Selenastrum capricornutum. 
Environmental Toxicology and Pharmacology, 2013, 35: 320-324.

[14] Lin Tao, Chen Yanqiu, Chen Wei. Toxic effect of sulfadiazine on the growth of zebrafish embryos in the water body. Journal of Safety and Environment, 2014, 14(3) : 324-327. [林涛, 陈燕秋, 陈卫. 水体中磺胺嘧啶对斑马鱼的生态毒性 效应. 安全与环境学报, 2014, 14(3): 324-327.]

[15] Jiang L, Hu XL, Yin DQ et al. Occurrence, distribution and seasonal variation of antibiotics in the Huangpu River, Shanghai, China. Chemosphere, 2011, 82(6): 822-828.

[16] Jia A, Wan Y, Xiao Y et al. Occurrence and fate of quinolone and fluoroquinolone antibiotics in a municipal sewage treatment plant. Water Research, 2012, 46(2) : 387-394.

[17] Le TX, Munekage Y. Residues of selected antibiotics in water and mud from shrimp ponds in mangrove areas in Viet Nam. Marine Pollution Bulletin, 2004, 49(11/12) : 922-929.

[18] Rodriguez-Mozaz S, Chamorro S, Marti E et al. Occurrence of antibiotics and antibiotic resistance genes in hospital and urban wastewaters and their impact on the receiving river. Water Research, 2015, 69: 234-242.

[19] Na GS, Gu J, Ge LK et al. Detection of 36 antibiotics in coastal waters using high performance liquid chromatography-tandem mass spectrometry. Chinese Journal of Oceanology and Limnology, 2011, 29(5) : 1093-1102.

[20] Ma Jing'an, Li Hongqing. Preliminary discussion on eutrophication status of lakes, reservoirs and rivers in china and overseas. Resources and Environment in the Yangtze Basin, 2002, (6) : 575-578. [马经安, 李红清. 浅谈国内外江河湖库水 体富营养化状况. 长江流域资源与环境, 2002, (6) : 575-578.]

[21] Tang Jun, Zhang Fuhai, Wang Chenchen et al. Investigation of sulfonamide antibiotics residue in the water of Chaohu Lake and its inlet rivers. Journal of Safety and Environment, 2014, (4) : 334-338. [ 唐俊, 张付海, 王晨晨等. 巢湖及入湖河 流中磺胺抗生素残留现状分析. 安全与环境学报, 2014, (4) : 334-338.]

[22] Lei XN, Lu JJ, Liu ZL et al. Concentration and distribution of antibiotics in water-sediment system of Bosten Lake, Xinjiang. Environmental Science and Pollution Research, 2015, 22(3) : 1670-1678.

[23] Lu Xinyu, Zhan Jian, Han Yulong. Nanchang Qingshanhu Lake water environment situation and repair advice. China Water Transport ( The second half month)，2015，(7)：146-147, 162. [卢辛宇, 詹健, 韩玉龙. 南昌青山湖水环境现状与 修复建议. 中国水运(下半月), 2015, (7): 146-147, 162.]

[24] Reviewing New Chemicals under the Toxic Substances Control Act[EB/OL]. http://www.epa.gov/oppt/newchems/tools/ 21 ecosar.htm.

[25] Zhou LJ, Ying GG, Liu S et al. Simultaneous determination of human and veterinary antibiotics in various environmental matrices by rapid resolution liquid chromatography-electrospray ionization tandem mass spectrometry. Journal of Chromatography A, 2012, 1244: 123-138.

[26] Ding Huijun, Wu Yixiao, Zhong Jiayou et al. Role of two mediators in sulfonamide antibiotics degradation by laccase oxidation system. China Environmental Science, 2016, (5) : 1469-1475. [ 丁惠君, 吴亦潇, 钟家有等. 两种介体物质在漆 酶降解磺胺类抗生素中的作用. 中国环境科学, 2016, (5): 1469-1475.]

[27] Ding HJ, Wu YX, Zou BC et al. Simultaneous removal and degradation characteristics of sulfonamide, tetracycline, and quinolone antibiotics by laccase-mediated oxidation coupled with soil adsorption. Journal of Hazardous Materials, 2016, 307: 350-358.

[28] Couperus NP, Pagsuyoin SA, Bragg LM et al. Occurrence, distribution, and sources of antimicrobials in a mixed-use watershed. Science of the Total Environment, 2016, 541: 1581-1591.

[29] Wang Liping, Zhang Mingkui, Zheng Shun'an. Adsorption-desorption characteristics and biological effects of enrofloxacin in agricultural soils. Chinese Journal of Soil Science, 2008, (2) : 393-397. [王丽平, 章明奎, 郑顺安. 土壤中恩诺沙星 的吸附-解吸特性和生物学效应. 土壤通报, 2008, (2) : 393-397.]

[30] Huang Lizhang, Jin Lahua, Wan Jinbao. Aixi Lake water pollution situation analysis and countermeasures. China's water conservancy institute of 2013 academic essays, S2, lakes development and protection. Water Conservancy Society of China, 2013: 5. [ 黄立章, 金腊华, 万金保. 艾溪湖水污染现状分析及治理对策.中国水利学会 2013 学术年会论文 集—S2 湖泊治理开发与保护. 中国水利学会, 2013: 5.]

[31] Feng Baojia, Zeng Qiang, Zhao Liang et al. Source Distribution of antibiotics in water environment and itsimpact on human health. Environmental Monitoring Management and Technology, 2013, (1): 14-17, 21. [冯宝佳, 曾强, 赵亮等. 水环 境中抗生素的来源分布及对健康的影响. 环境监测管理与技术, 2013, (1): 14-17, 21.] 
[32] Chen Yang. Study on relationship between water eutrophication and environmental factors in Yao Lake[Dissertation]. Nanchang: East China Jiaotong University, 2012. [陈杨. 瑶湖水体营养化现状及其与环境因子关系的研究 [ 学位论文]. 南昌: 华东交通大学, 2012.]

[33] Tang Zehua. Influence study on the classical garden from urbanization: Taking the Xianghu park in Nanchang City for example[Dissertation]. Nanchang: Jiangxi Agricultural University,2012. [汤泽华. 城市化对古典园林的影响研究一一 南昌象湖公园为例 [学位论文]. 南昌: 江西农业大学, 2012.]

[34] Alexy R, Kumpel T, Kummerer K. Assessment of degradation of 18 antibiotics in the Closed Bottle Test. Chemosphere, 2004, 57: 505-512.

[35] Murata A, Takada H, Mutoh K et al. Nationwide monitoring of selected antibiotics: Distribution and sources of sulfonamides, trimethoprim, and macrolides in Japanese rivers. Science of the Total Environment, 2011, 409(24) : 5305-5312.

[36] Xu J, Zhang Y, Zhou CB et al. Distribution, sources and composition of antibiotics in sediment, overlying water and pore water from Taihu Lake, China. Science of the Total Environment, 2014, 497: 267-273.

[37] Li WH, Shi Y, Gao LH et al. Occurrence of antibiotics in water, sediments, aquatic plants, and animals from Baiyangdian Lake in North China. Chemosphere, 2012, 89(11): 1307-1315.

[39] Commission E. Commission Technical Guidance Document in Support of Commission Directive 93//67/EEC on Risk Assessment for New Notified Substances and Commission Regulation (EC) no. 1488/94 on Risk Assessment for Existing Substance, Part II, 2003.

[39] Lei Bingli, Huang Shengbiao, Wang Zijian. Theories and methods of ecological risk assessment. Progress in Chemistry, 2009, 21: 350-358. [雷炳莉, 黄圣彪, 王子健. 生态风险评价理论和方法. 化学进展, 2009, 21: 350-358.]

[40] U.S. EPA, 2011. U.S. EPA Website, <http://www.epa.gov/oppt/newchems/tools/21 ecosar.htm>.

[41] Yan C, Yang Y, Zhou J et al. Antibiotics in the surface water of the Yangtze Estuary: Occurrence, distribution and risk assessment. Environ Pollut, 2013, 175: 22-29.

[42] Hernando MD, Mezcua M, Fernandez-Alba AR et al. Environmental risk assessment of pharmaceutical residues in wastewater effluents, surface waters and sediments. Talanta, 2006, 69(2) : 334-342. 